\title{
An instance-based scoring system for indoor landmark salience evaluation
}

\author{
LITAO ZHU ${ }^{1,2,3}$, HANA ŠVEDOVÁ ${ }^{4}$, JIE SHEN ${ }^{1,2,3}$, ZDENĚK STACHOŇ $^{4}$, \\ JIAFENG SHI ${ }^{1,2,3}$, DAJANA SNOPKOVÁ ${ }^{4}, X^{\prime A O ~ L I} I^{1,2,3}$
}

1 Key Laboratory of Virtual Geographic Environment, Nanjing Normal University, Ministry of Education, Nanjing, China; e-mail: Litao.Zhuu@outlook.com, jiafeng.shi@outlook.com, xiaoliim@outlook.com

2 Jiangsu Center for Collaborative Innovation in Geographical Information Resource Development and Application, Nanjing, China

3 School of Geography, Nanjing Normal University, Nanjing, China; e-mail: shenjie@njnu.edu.cn

${ }^{4}$ Masaryk University, Faculty of Science, Department of Geography, Brno, Czechia; e-mail: zstachon@geogr.muni.cz

ABSTRACT Navigation in a complex indoor environment can be difficult, and pedestrians may find themselves disoriented. As the featured objects of an environment, indoor landmarks play an important role in navigation. A review of the existing literature in outdoor landmark evaluation methods, however, shows that they cannot be fully applicable in any indoor environment. In this paper, an instance-based scoring system is proposed for analyzing the indicators that influence the salience of spatial objects from visual, semantic and structural aspects. An Analytic Hierarchy Process was applied to calculate landmark weights using these indicators. Two types of indoor scenes were employed as instances to verify the validity of this method, the Dongchenghui shopping mall (Nanjing, China) using a subjective questionnaire and interview method, and the headquarters of Masaryk University (Brno, Czechia) using an objective eyetracking method. The result of the two instances showed that the proposed method was feasible.

KEY WORDS indoor landmark - landmark salience - scoring system - analytic hierarchy process - eye-tracking

ZHU, L., ŠVEDOVÁ, H., SHEN, J., STACHOŇ, Z., SHI, J., SNOPKOVÁ, D., LI, X. (2019): An instance-based scoring system for indoor landmark salience evaluation. Geografie, 124, 2, 103-131.

Received November 2018, accepted April 2019.

(C) Česká geografická společnost, z. s., 2019 


\section{Introduction}

People spend around $80 \%$ of their time in an indoor environment (Klepeis et al. 2001). Landmarks are fundamental elements in human wayfinding strategies and should be integrated into navigation services (Peters, Wu, Winter 2010). Landmarks can also serve as spatial reference points (Lynch 1960; Presson, Montello 1988), cognitive anchors (Golledge 1999), and a means for orientation, wayfinding and spatial communication (Richter, Winter 2014). Landmarks are more cognitively salient and prominent than the surrounding spatial features (Sorrows, Hirtle 1999). These salient objects are easily recognized and remembered (Millonig, Schechtner 2007) and allow people to more easily define the locations of other features and judge their own location (Zhang, Li, Fang 2010). People also prefer using landmarks in route guidance because these objects are more understandable and elicit better results than other route guidance methods (Sorrows, Hirtle 1999; Raubal, Winter 2002).

Spatial structures in indoor and outdoor environments have differences (Lorenz, Ohlbach, Stoffel 2006; Hölscher, Brösamle 2007). The attributes of indoor landmarks during navigation are significantly different from outdoor landmarks, for example, in their physical features or function. The methods applied in selecting outdoor landmarks cannot be directly applied indoors.

Exploring suitable indicators for evaluating and extracting indoor landmarks for use in providing navigation instructions is important in designing locationbased services that are immediate and meaningful. A method for evaluating indoor landmark salience in order to extract the most salient spatial features in an area and help organize route guidance messages is required. Indoor landmarks play an important role in navigation. Some literature has studied the methods for evaluating indoor landmark salience (Lyu, Yu, Meng 2015; Fellner, Huang, Gartner 2017; Li et al. 2017).

The paper proposes an instance-based scoring system as method of evaluating indoor landmark salience. Using an Analytic Hierarchy Process (AHP), the method calculates the reasonable landmark weights that agree with user perceptions. To verify this method, we applied it practically to the Dongchenghui shopping mall near Nanjing Normal University (Nanjing, China) and the headquarters of Masaryk University (Brno, Czechia).

The paper is organized as follows. Section 2 describes the related work, namely, the outdoor and indoor landmark salience evaluation method. Section 3 introduces the instance-based scoring system for extracting and evaluating indoor landmark salience. Section 4 describes the first experiment at the Dongchenghui shopping mall (China) to evaluate landmark salience using a qualitative analysis of questionnaires and interviews. Section 5 describes the second experiment at the headquarters of Masaryk University (Brno, Czechia) to evaluate landmark 
salience evaluation using eye-tracking. A discussion follows in Section 6. The article is concluded in Section 7 with suggestions for future work.

\section{Related work}

\subsection{Landmarks in navigation}

Humans today frequently need to find their way through indoor environments such as shopping malls, universities, hospitals, etc. In many cases, people find it difficult to perform wayfinding tasks in an unfamiliar environment (Raubal 2001). The main reason for environments being too complex to facilitate wayfinding is a deficiency of clues (Raubal, Egenhofer 1998). Landmarks play a fundamental role in human orientation and navigation. The concept of a landmark was proposed by Lynch (1960), who considered landmarks as identifiable objects, that serve as external reference points. Similar findings were made by Siegel and White (1975), who suggested that landmarks can be defined as the prominent features in an environment that are unique or contrast with the surroundings. Golledge (1999) observed human wayfinding behavior and found that the people select route criteria that are most frequently linked to landmarks. As meaningful features for human navigation, landmarks located both outdoors and indoors serve identical roles (Sorrows, Hirtle 1999; Yang, Worboys 2011). Anything prominent in a scene can be a landmark (Presson, Montello 1988). This notion can be applied outdoors or indoors, however, indoor and outdoor navigation are significantly different because of the differences in topological structures (Lorenz, Ohlbach, Stoffel 2006). Indoor landmarks are also navigational aids and more important than outdoor landmarks, as people are more likely to lose their way indoors than outside (Radoczky 2003; Huang, Gartner 2010). Li et al. (2017) provided a landmark-based cognition strength grid model for improving indoor path planning and guidance. Russo, Zlatanova, Clementini (2014) proposed an algorithm to automatically generate indoor route instructions that included visible landmarks (doors, windows, passages). Similarly, Fellner, Huang, Gartner (2017) provided a category-based method to generate indoor landmark-based route instructions for navigation.

Klippel and Winter (2005) summarized the characteristics of landmarks as: (1) Any object prominent in the surroundings can be a landmark; (2) An intersection of roads lacking explicit semantics can also be referred to as a landmark in certain cases; (3) Landmarks have an important role in wayfinding; (4) Landmarks are remembered at the beginning of the formation of spatial knowledge, namely landmark knowledge; (5) Landmarks can be used as "anchor points" to build environmental knowledge; (6) Landmarks can convey route knowledge in the form of words and graphics; (7) The degree of integration of landmarks on a road is 
different; (8) When direction needs to be changed, the landmarks of street intersections (decision points) are more relevant; (9) Landmarks are generally better at assisting users in wayfinding than street signs.

\subsection{Landmark salience estimation}

Landmark salience estimation is one common evaluation and extraction method. The degree of landmark salience is primarily measured by its visual, semantic and structural aspects (Sorrows, Hirtle 1999; Raubal, Winter 2002; Caduff, Timpf 2008). Visual salience refers to spatial features noticeable because of their visual attraction, such as colour, size or form. Semantic salience refers to the significant meaning and function of spatial features. Structural salience explains the important location of spatial features. Sorrows and Hirtle (1999) proposed the three visual, cognitive and structural indicators of landmarks. Raubal and Winter (2002) developed a formal model with these indicators that aimed to capture the perceptual and cognitive aspects of objects which were then used to calculate landmark salience. Nothegger, Winter, Raubal (2004) measured the objects for facade area, shape, colour, visibility and semantic attraction in selecting the salient features for route directions. Klippel and Winter (2005) extended the structural salience model for selecting landmarks at intersections most suited to indicating a change in direction (e.g., turn left).

Several functions have been extended for measuring landmark salience according to visual, semantic and structural features. Elias (2003) proposed using the classification method ID3 (supervised classification algorithm) and running machine learning methods to identify potential landmark candidates for existing topographic or cadastral data sets. Similarly, Winter et al. (2008) proposed a model for building machine generated hierarchies of landmarks from salience that could distinguish between local and global landmarks. Duckham, Winter, Robinson (2010) explored a category-based scoring system and landmark weighting in order to identify landmarks. This method established a ranked order of different categories (e.g., restaurants, filling stations or schools), which is based on nine different aspects (e.g. physical size, prominence, ubiquity and familiarity, length of description, spatial extents, or permanence). These studies contributed to both landmark salience theory and its application. Most of the salience theory for landmark identification and extraction are limited to the outdoors, whereas landmarks for indoor navigation have been neglected. 


\subsection{Indoor landmark salience}

With the development of indoor positioning, navigation, maps and other related research, indoor landmark salience has been looked at by researchers but is still in a preliminary stage. Millonig, Schechtner (2007) proposed a method to introduce landmark-based pedestrian navigation systems via mobile phones at a major train station, concentrating on identifying the visibility attribute of salient indoor landmarks. They extended the definition of landmarks so that any object could be considered a landmark if it were distinguishable enough from adjacent items. Later, Lyu, Yu, Meng (2015) proposed indoor landmark salience indicators as a basis for describing visual accessibility and shape perceivability (from visual salience), accessibility and location importance (from structural salience) and functional importance (from semantic salience), using them to create a computational indoor landmark extraction method. Ohm, Müller, Ludwig (2015) identified landmarks in indoor environments using eye-tracking and took into account four categories: architecture (e.g., pillars), function (e.g., doors, stairs, and elevators), information (e.g., signs and posters) and furniture (e.g., tables). They showed that "functional" salient objects such as stairs and doors are suitable for indoor pedestrian navigation. Hund (2016) demonstrated through cognitive experiments that visuospatial working memory plays an important role in wayfinding and giving directions in an indoor environment. Fellner, Huang, Gartner (2017) proposed a category-based method adapted from Duckham, Winter, Robinson (2010) for scoring landmark suitability factors in indoor navigation and used it to generate landmark-based route instructions to support people's wayfinding activities in unfamiliar indoor environments. In order to determine the salient properties of indoor landmarks, $\mathrm{Li}$ et al. (2017) used the questionnaires and determined four new characteristics (utility, functional importance, referenced rate, entrances or exits) that could be introduced to indoor landmark categories.

In summary, landmarks have an important enhancing effect on navigation systems. Current methods in extracting landmarks primarily target applications to aid navigation but tend to remain at a qualitative discussion level in terms of representing spatial knowledge and neglect the user's subjective experience. Most of these methods are limited to the outdoors. Providing a method for selecting indoor landmarks is necessary in order to improve user satisfaction in navigation. Based on previous studies, this paper proposes an instance-based scoring system that allows users to identify and evaluate indoor landmarks by themselves. 


\section{Method}

\subsection{Landmark suitability indicators and scoring}

The first method in identifying landmarks was presented by Raubal and Winter (2002). This method relies on instance-level data about the visual, semantic and structural properties of individual spatial features, which are then used to calculate outdoor landmark salience. Duckham, Winter, Robinson (2010) proposed a different category-based method for outdoor navigation that relies on the commonly available data of categorized points of interest. Similarly, Fellner, Huang, Gartner (2017) applied this category-based method for indoor navigation. As illustrated by the literature, a distinction is made between methods in identifying individual objects (instances) or categories of objects (types). However, the problem in an indoor environment is the lack of a consistent and uniform method for classifying indoor landmarks. Multi-varied indoor scenes need effective individual evaluation procedures, but there is less individualized evaluation methodology available. This study is based on previous studies and proposes an instance-based scoring system. A preliminary individual evaluation method is constructed to provide a means for quantifying indoor landmark salience evaluation. The indicators of indoor landmark salience are adapted from Duckham, Winter, Robinson (2010), Fellner, Huang, Gartner (2017), Lyu, Yu, Meng (2015). The formal model for landmark salience includes three visual indicators:

1. Physical size: larger spatial features are more easily seen and better landmark candidates than smaller features. The value of physical size is measured using the Likert scale. For example, a rubbish bin with a smaller visible area is assigned a score of 1 , while a shop with a larger visible area is assigned 5.

2. Prominence: spatial features are visually prominent and better landmark candidate than those with few distinguishing features. The degree of prominence is often related to factors such as colour, lighting, etc. The Likert five-level scale was suitable for evaluating this value.

3. Availability of a unique label: spatial features with a unique and visible label that can be used as a reference in route instructions are better landmark candidates than those without. The indicator is assigned three levels: "no label", "labelled" and "unique label", indicated by 1, 2 or 3 respectively.

Semantic salience is measured according to three properties:

1. Familiarity: spatial features that are familiar are better landmark candidates. For example, the familiarity of a shop is mainly associated with its brand.

2. Length of description: spatial features that require shorter descriptions are better landmark candidates than features that require long and complex 
descriptions. This indicator is assigned three levels: "without modifiers", "single word modifiers" and "other", indicated by 3, 2 and 1, respectively.

3. Uniqueness: spatial features that are unique are better landmark candidates. If a landmark appears more than once in the same indoor environment, its semantic significance is reduced. A unique landmark in the same indoor environment is assigned 1 , the opposite value being 0 .

Structural salience consists of three properties:

1. Spatial extent: point-based spatial features are likely to be more suitable landmarks, as they are less ambiguous than features with spatial extent. A point-based landmark is therefore assigned 1 and a polygon-based landmark is assigned 0.

2. Permanent: spatial features that are expected to change less frequently are better candidate landmarks. A permanent landmark is assigned 1 and other are assigned 0.

3. Location importance: spatial features are important locations that are better landmark candidates. Spatial features are located on or near routes or intersections (such as staircases) and have a higher structural salience (Table 1).

The values should be normalized with feature scaling because the indicators use different scoring methods. All values are therefore brought into the range $[0,1]$ as follows:

$$
S_{f}=\frac{\operatorname{score}(i)-\min (\{\operatorname{score}(i) \mid i \in I\})}{\max (\{\operatorname{score}(i) \mid i \in I\})-\min (\{\operatorname{score}(i) \mid i \in I\})}
$$

where score $(i)$ is an original value, $S_{f}$ is the normalized value, $i$ is a landmark, $I$ is a set $\{a, \beta, \gamma, \delta, \zeta, \eta, \vartheta, l, k\}$ of all landmark indicators, $\max (\{\operatorname{score}(i) \mid i \in I\})$ refers

Table 1 - Indicators and indoor landmark salience measurements

\begin{tabular}{llll}
\hline Type & Indicator & Measurement & Degree of Attractiveness \\
\hline Visual & Physical size $(\alpha)$ & $\alpha \in\{1,2,3,4,5\}$ & $S_{\text {vis }}=w_{\alpha} S_{\alpha}+w_{\beta} S_{\beta}+w_{\gamma} S_{\gamma}$ \\
& Prominence $(\beta)$ & $\beta \in\{1,2,3,4,5\}$ & \\
& Availability of a unique label $(\gamma)$ & $\gamma \in\{1,2,3\}$ & \\
\hline Semantic & Familiarity $(\delta)$ & $\delta \in\{1,2,3,4,5\}$ & $S_{\text {sem }}=w_{\delta} S_{\delta}+w_{\zeta} S_{\zeta}+w_{\eta} S_{\eta}$ \\
& Description length $(\zeta)$ & $\zeta \in\{1,2,3\}$ & \\
& Uniqueness $(\eta)$ & $\eta \in\{\mathrm{T}: 1, \mathrm{~F}: 0\}$ & \\
\hline Structural & Spatial extent $(\vartheta)$ & $\vartheta \in\{\mathrm{T}: 1, \mathrm{~F}: 0\}$ & $\mathrm{S}_{\text {str }}=w_{\vartheta} S_{\vartheta}+w_{\iota} S_{\iota}+w_{\kappa} S_{\kappa}$ \\
& Permanence $(\iota)$ & $\iota \in\{\mathrm{T}: 1, \mathrm{~F}: 0\}$ & \\
& Location importance $(\kappa)$ & $\kappa \in\{1,2,3\}$ & \\
& & & \\
\hline
\end{tabular}


to the maximum value of the subjects and $\min (\{\operatorname{score}(i) \mid i \in I\})$ is the minimum scoring value of the subjects.

\subsection{Landmark Weight Estimation}

It is extremely difficult to assign relative weights to the different criteria involved in estimating the suitability of indoor landmarks for navigation. We therefore suggest using a method such as the Analytical Hierarchy Process (AHP) that allows weights to be estimated. The AHP is a method for systematic and structured analysis of complex decision-making processes (Saaty 1994). Because it is difficult to determine the relative importance of landmark weights for complex indoor navigation problems, the AHP can reduce the complexity of decisions into a series of pairwise comparisons between competing attributes. To compute indoor landmark salience models, the AHP has three basic steps as follows.

Step 1: Determine the goal and select the indicators. It begins by decomposing the overall goal into a number of criteria and sub-criteria. The goal is to compute the weights of indoor landmark salience that represent the top level of the hierarchy. The criterion layer consisting of three indicators represents the second level of the hierarchy and the sub-criterion layer consisting of nine indicators is the third level (Fig. 1).

Step 2: Use a questionnaire to collect expert opinions. The questionnaire was designed to make all possible pair-wise comparisons between factors. A comparison of two criteria determines the more preferable criterion, its level of importance

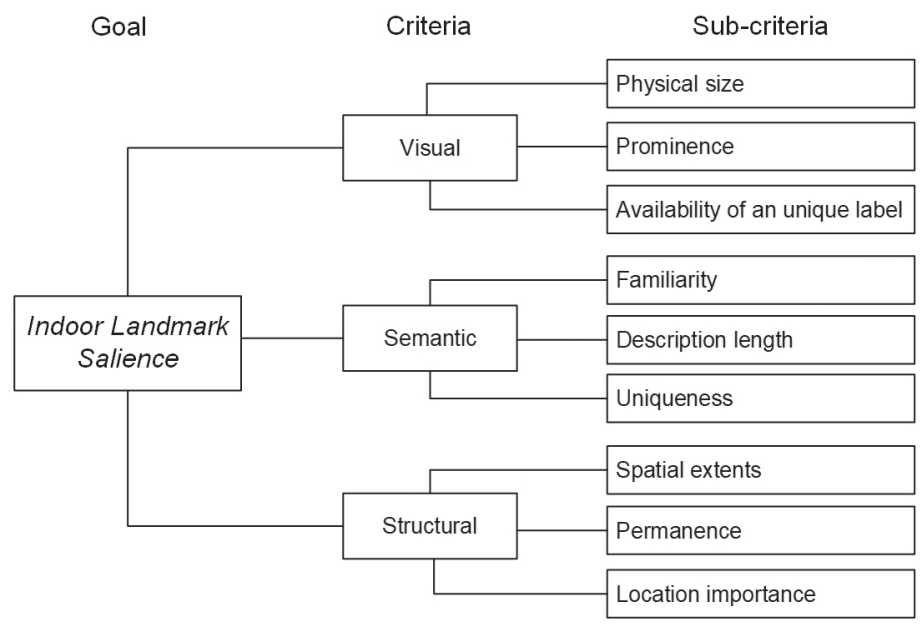

Fig. 1 - Indoor landmark salience hierarchy model 
and by how much it more is preferable. A typical nine-point scale shows values from the set $\{1 / 9,1 / 8,1 / 7,1 / 6,1 / 5,1 / 4,1 / 3,1 / 2,1,2,3,4,5,6,7,8,9\}$ in an AHP questionnaire, the minimum value $1 / 9$ representing the least important, 1 representing equal importance and 9 representing the most important (Saaty 1994).

Step 3: Test the consistency. The Consistency Index (CI) is used to express the degree of consistency.

$$
C I=\frac{\lambda_{\max }-n}{n-1}
$$

where $\lambda_{\max }$ is the maximum eigenvalue of the matrix of importance ratios and $n$ is the number of factors. Accordingly, the Constituency Ratio $(C R)$ is given by:

$$
C R=\frac{C I}{R I}
$$

If the value of the consistency ratio $C R$ is less than or equal to 0.1 the questionnaire is considered acceptable. If $C R$ is greater than 0.1, the questionnaire fails.

\subsection{Overall Suitability Score}

With the weights determined from the AHP, the overall salience of the landmark can be calculated as follows.

$$
S_{\text {sum }}=w_{\text {vis }} S_{\text {vis }}+w_{\text {sem }} S_{\text {sem }}+w_{\text {str }} S_{\text {str }}
$$

where $S_{v i s}$ is the scoring value of visual salience, $S_{\text {sem }}$ is the scoring value of semantic salience, $S_{s t r}$ is the scoring value of structural salience and the range $w_{\alpha}$ to $w_{x}$ refers to the weights of the sub-criteria consisting of nine indicators.

In order to verify the validity of the method of the instance-based scoring system, two types of indoor scenes were employed as instances. Experiment 1 in the following section applied the subjective questionnaire method with participants at the Dongchenghui shopping mall (Nanjing, China).

\subsection{Eye-tracking}

Eye-tracking is a frequently used method for collecting data in user studies in order to evaluate navigation using both outdoor and indoor landmarks. Eye-tracking is fundamentally the measurement of eye activity. Movements of the human eye are not continuous but a sequence of fixations and saccades. Researchers have made much effort over the years to learn how human spatial thinking functions and to justify human decisions and strategies during orientation (Kato, Takeuchi 
2003). Sight is one of the main senses that influences our decisions, and based on hypothesis, sight is strongly connected to thinking. An instant visual perception can indicate what people may be thinking about and what is therefore prevalent in their cognitive processes (Just, Carpenter 1976). One of the main problems with and disadvantages of eye-trackers is their inability to measure and evaluate peripheral vision. Users perceive a certain amount of information from peripheral vision that is not included in data represented by fixations and saccades. Another common problem is landmarks used only as sight anchors when users focus on landmarks but do not obtain any valuable information useful for orientation (Van Gog et al. 2009). This method was used in Experiment 2.

\section{Experiment 1}

This experiment analysed the study area using the scoring system method for indoor landmarks and an AHP to calculate the weight of each category for overall landmark evaluation.

\subsection{Participants}

Six subjects (three females, three males, mean age of $22 \pm 1$ years) participated in the experiment. All subjects were postgraduates in the field of cartography and geographic information systems at the Nanjing Normal University (China). All participants were also very familiar with the study area and they had been there at least five times.

\subsection{Study area}

The study area was the Basement 1 (B1) of the Dongchenghui shopping mall near Nanjing Normal University (Nanjing, China). The Dongchenghui shopping mall is a commercial complex with businesses, shops and entertainment, social and recreational areas and other types of complex functions. This kind of multifunctional composition makes it difficult for visitors to find efficient paths to reach their destinations. There are many types of landmarks along the path (Fig. 2). It is a typical experimental area that can be used to verify the validity of the instancebased scoring system method for indoor landmark salience evaluation. 


\subsection{Landmark selection}

The participants were instructed to follow the route marked according to the study area. When they completed this route, their oral reports in which they described the objects along this route in as much detail as possible were collected. After summarizing the collected data, the results consisted of fifty selected indoor objects. Some of the potential landmark candidates identified were "East Side Cross Station", "Orange Iron Box 2", "Washroom", "Escalator 4", and "Indoor Map Guide Sign 2" (Fig. 2).

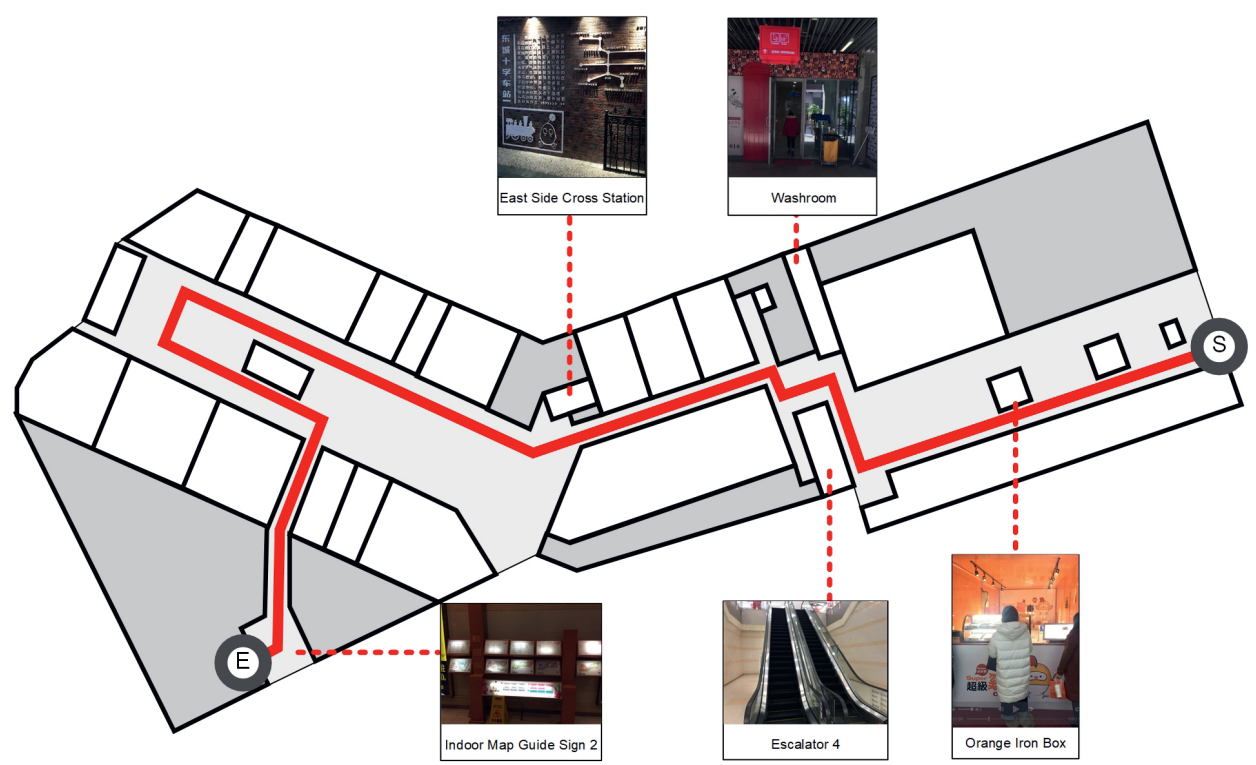

Fig. 2 - Study area and example of five landmarks in the Dongchenghui shopping mall basement (B1)

Fig. 3 - Statistical distribution of landmark categories

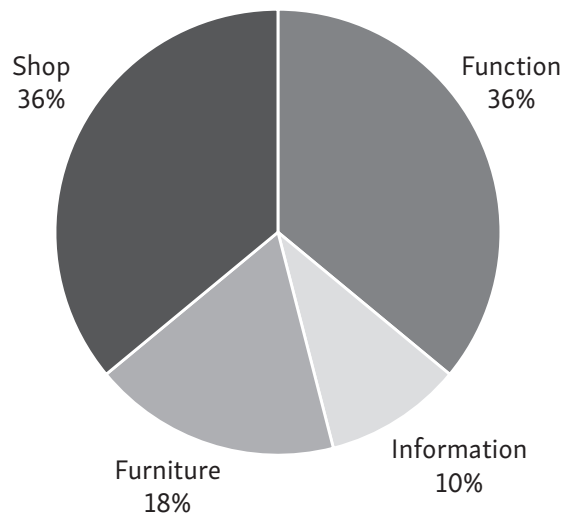


The fifty selected objects were assigned into four landmark categories (Fig. 3): shops (e.g., restaurants, clothing stores), function (e.g., stairs, elevators, doors), furniture (e.g., sculptures, chairs, benches) and information (e.g., posters, indoor map guides). Because the Dongchenghui is a commercial complex, most of the selected objects were shops (36\%) and function elements (36\%).

\subsection{Calculating landmark salience and weighting}

\subsubsection{Evaluation indicators of indoor landmark salience}

The evaluation of landmark salience based on the method proposed above was performed by six participants. All had a proper understanding of the concepts and indicators of landmark salience. The scores of each indicator for each landmark example are shown in Table 2.

Because the scoring ranges of landmark salience evaluation indexes were not uniform, the eigenvalues were normalized using the scaling method feature in Equation (1). Table 2 shows the value of different landmark indicators scored by participants as $\alpha, \theta, \gamma, \delta, \zeta, \eta, \theta, \iota, \kappa$ and these results as $S_{\alpha}, S_{\theta}, S_{\gamma}, S_{\delta}, S_{\zeta}, S_{\eta}, S_{\theta}, S_{\iota}, S_{\varkappa}$ after normalizing. Five landmarks are given as examples.

\subsubsection{Calculation of indicator weights of at each level}

Indoor landmark salience is a multi-indicator comprehensive evaluation. Determination of the weight coefficient is crucial to the evaluation result.

In order to deal with this problem, an AHP was introduced to evaluate indoor landmark salience and replace expert scoring in order to calculate indicator weight. The AHP is especially suitable for cases with few sample data and where the evaluation target structure is complicated. First, we built a pairwise comparison matrix of $n \times n$, where $n$ was found by comparing each pair of factors. We used a matrix of landmark salience factors. An example question may be "Which

Table 2 - The participants' initial and normalized average scores

\begin{tabular}{|c|c|c|c|c|c|c|c|c|c|c|c|c|c|c|c|c|c|c|}
\hline \multirow[t]{2}{*}{ Landmark Name } & \multicolumn{6}{|c|}{ Visual } & \multicolumn{6}{|c|}{ Semantic } & \multicolumn{6}{|c|}{ Structural } \\
\hline & $a$ & $\mathrm{~S}_{\alpha}$ & $\beta$ & $S_{\beta}$ & $\gamma$ & $S_{\gamma}$ & $\delta$ & $\mathrm{S}_{\delta}$ & $\zeta$ & $\mathrm{S}_{\zeta}$ & $\eta$ & $S_{\eta}$ & $\vartheta$ & $S_{\vartheta}$ & $\iota$ & $S_{t}$ & k & $S_{k}$ \\
\hline East Side Cross Station & 4 & 0.75 & 5 & 1 & 3 & 1 & 2 & 0.25 & 2 & 0.5 & 1 & 1 & 1 & 1 & 1 & 1 & 2 & 0.5 \\
\hline Orange Iron Box 2 & 3 & 0.5 & 4 & 0.75 & 3 & 1 & 3 & 0.5 & 1 & 0 & 0 & 0 & 1 & 1 & 0 & 0 & 2 & 0.5 \\
\hline Washroom & 3 & 0.5 & 3 & 0.5 & 3 & 1 & 5 & 1 & 3 & 1 & 0 & 0 & 1 & 1 & 1 & 1 & 3 & 1 \\
\hline Escalator 4 & 4 & 0.75 & 5 & 1 & 1 & 0 & 5 & 1 & 3 & 1 & 0 & 0 & 1 & 1 & 1 & 1 & 3 & 1 \\
\hline Indoor Map Guide Sign 2 & 4 & 0.75 & 3 & 0.5 & 2 & 0.5 & 2 & 0.25 & 2 & 0.5 & 0 & 0 & 1 & 1 & 1 & 1 & 2 & 0.5 \\
\hline
\end{tabular}


factor do you feel is important in indoor landmark salience - visual or semantic?" An expert would select the values from the set $\{1 / 9,1 / 8,1 / 7,1 / 6,1 / 5,1 / 4,1 / 3,1 / 2$, $1,2,3,4,5,6,7,8,9\}$ according to their spatial knowledge. The selected number is then recorded in the matrix component according to their answer. The landmark weights in the AHP model were obtained by computing the constructed judgment matrix (Table 3). The proportion of landmark salience from high to low is shown in Table 4 as: Prominence $(\beta)$ - Location importance $(\kappa)$ - Availability of a unique

Table 3 - Results of the judgment matrix in the AHP

\begin{tabular}{|c|c|c|c|c|}
\hline$S_{\text {sum }}$ & Svis & $S_{\text {sem }}$ & $S_{\text {str }}$ & $w_{i}$ \\
\hline Svis & 1.0000 & 3.0000 & 2.0000 & 0.5396 \\
\hline$S_{\text {sem }}$ & 0.3333 & 1.0000 & 0.5000 & 0.1634 \\
\hline$S_{\text {str }}$ & 0.5000 & 2.0000 & 1.0000 & 0.2970 \\
\hline \multicolumn{5}{|c|}{$\mathrm{Cl}=0.0088 ; \lambda_{\max }=3.0092$} \\
\hline Svis & a & $\beta$ & $\gamma$ & $w_{i}$ \\
\hline$a$ & 1.0000 & 0.2000 & 0.3333 & 0.1047 \\
\hline$\beta$ & 5.0000 & 1.0000 & 3.0000 & 0.6370 \\
\hline$\gamma$ & 3.0000 & 0.3333 & 1.0000 & 0.2583 \\
\hline \multicolumn{5}{|c|}{$\mathrm{Cl}=0.0370 ; \lambda_{\max }=3.0385$} \\
\hline$S_{\text {sem }}$ & $\delta$ & $\zeta$ & $\eta$ & $w_{i}$ \\
\hline$\delta$ & 1.0000 & 3.0000 & 0.3333 & 0.2499 \\
\hline$\zeta$ & 0.3333 & 1.0000 & 0.1667 & 0.0953 \\
\hline$\eta$ & 3.0000 & 6.0000 & 1.0000 & 0.6548 \\
\hline \multicolumn{5}{|c|}{$\mathrm{Cl}=0.0176 ; \lambda_{\max }=3.0183$} \\
\hline$S_{\text {str }}$ & $\vartheta$ & $\mathrm{\imath}$ & k & $w_{i}$ \\
\hline$\vartheta$ & 1.0000 & 0.3333 & 0.1429 & 0.0879 \\
\hline$\iota$ & 3.0000 & 1.0000 & 0.3333 & 0.2426 \\
\hline$\kappa$ & 7.0000 & 3.0000 & 1.0000 & 0.6694 \\
\hline
\end{tabular}

Table 4 - Results of the landmark weights

\begin{tabular}{|c|c|c|c|c|}
\hline Level 1 & & Level 2 & & Overall weight \\
\hline Criteria & $\mathrm{w}_{1}$ & Sub-criteria & $w_{2}$ & $\mathrm{~W}_{\mathrm{i}}=\mathrm{W}_{1} \mathrm{~W}_{2}$ \\
\hline \multirow[t]{3}{*}{ Visual } & 0.5396 & Physical size $(\alpha)$ & 0.1047 & 0.0565 \\
\hline & & Prominence $(\beta)$ & 0.6370 & 0.3437 \\
\hline & & Availability of a unique label $(\gamma)$ & 0.2583 & 0.1394 \\
\hline \multirow[t]{3}{*}{ Semantic } & 0.1634 & Familiarity $(\delta)$ & 0.6370 & 0.1070 \\
\hline & & 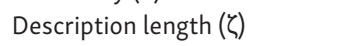 & 0.2583 & 0.0156 \\
\hline & & Uniqueness $(\eta)$ & 0.6370 & 0.0408 \\
\hline \multirow[t]{3}{*}{ Structural } & 0.2970 & Spatial extent (७) & 0.0879 & 0.0261 \\
\hline & & Permanence $(\iota)$ & 0.2426 & 0.0721 \\
\hline & & Location importance $(\kappa)$ & 0.6694 & 0.1988 \\
\hline
\end{tabular}


Table 5 - Overall landmark salience calculated for the example landmarks

\begin{tabular}{lcccccccccc}
\hline Landmark Name & $w_{\alpha} S_{\alpha}$ & $w_{\beta} S_{\beta}$ & $w_{\gamma} S_{\gamma}$ & $w_{\delta} S_{\delta}$ & $w_{\zeta} S_{\zeta}$ & $w_{\eta} S_{\eta}$ & $w_{\vartheta} S_{\vartheta}$ & $w_{\iota} S_{\iota}$ & $w_{\kappa} S_{\kappa}$ & $S_{\text {sum }}$ \\
\hline East Side Cross Station & 0.04 & 0.34 & 0.14 & 0.03 & 0.02 & 0.02 & 0.03 & 0.07 & 0.10 & 0.79 \\
Orange Iron Box 2 & 0.03 & 0.26 & 0.14 & 0.05 & 0.00 & 0.00 & 0.03 & 0.00 & 0.10 & 0.60 \\
Washroom & 0.03 & 0.17 & 0.14 & 0.11 & 0.00 & 0.04 & 0.03 & 0.07 & 0.20 & 0.78 \\
Escalator 4 & 0.04 & 0.34 & 0.00 & 0.11 & 0.00 & 0.04 & 0.03 & 0.07 & 0.20 & 0.83 \\
Indoor Map Guide Sign 2 & 0.04 & 0.17 & 0.07 & 0.03 & 0.00 & 0.02 & 0.03 & 0.07 & 0.10 & 0.53 \\
\hline
\end{tabular}

label $(\gamma)$ - Familiarity $(\delta)$ - Permanent $(\iota)$ - Physical size $(\alpha)$ - Uniqueness $(\eta)$ -

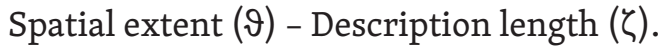

\subsection{Results}

After multiplying the normalized scores and weights, the results of the overall landmark salience evaluation were obtained according to the Equation (4; Table 5).

The fifty selected landmarks were categorized according to four types: shops, information, function, and furniture. The results of the computing overall landmark salience for indoor landmark categories are given in Figure 4. Shop landmarks had the highest overall landmark salience, followed by function, furniture, then information. This agrees with the experimental results of Ohm, Müller, Ludwig (2015), that functional landmarks (e.g., elevators, doors) play an important role in wayfinding. However, we have distinguishable differences from the indoor scenario. Because the experiment was conducted in a shopping mall, the result of computing the shop landmark salience was the highest value. Furthermore, the method also needs verifying by experiment whether individuals demonstrate spatial cognition of the study area according to the users' cognitive preferences.

Fig. 4 - Overall landmark salience for each category

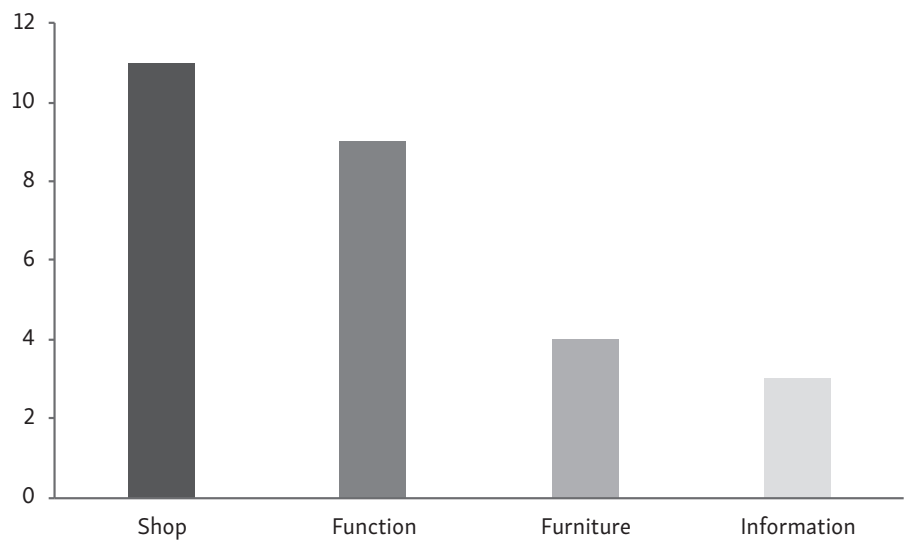




\subsection{Discussion}

Landmark salience was evaluated in this study area according to a proposed instance-based scoring system method. One major factor in the feasibility of this method is whether it satisfied the users' spatial cognition. The Likert scale and Pearson correlation coefficient were also used to compare the consistency of the computed landmark salience results and the results of users' spatial cognition.

Thirty random visitors to the experimental area (13 males and 17 females) were selected as participants. The participants were asked to indicate their level of agreement with landmark salience according to a declarative statement. On a five-point Likert scale, for example, each point can be assigned an agreement level: Strongly disagree - 1; Disagree - 2; Neither agree nor disagree - 3; Agree - 4 and Strongly agree -5 (Fig. 5). Simple statements such as "This spatial feature is useful for navigating" were given with pictures of the fifty selected landmarks on a mobile phone screen using the Likert scaling method.

The Pearson correlation coefficient was used to calculate the correlation between the indoor landmark salience evaluation result and the participants' scoring results. In statistics, the Pearson's correlation coefficient is a measure of the linear correlation between two variables $X$ and $Y$.

$$
r=\frac{n \sum X Y-\sum X \times \sum Y}{\sqrt{\left[n \sum X^{2}-\left(\sum X\right)^{2}\right] \times\left[n \sum Y^{2}-\left(\sum Y\right)^{2}\right]}}
$$

where is a value between 1 and -1 such that 1 is a total positive linear correlation, 0 is no linear correlation and -1 is a total negative linear correlation.

Fig. 5 - Likert scale method

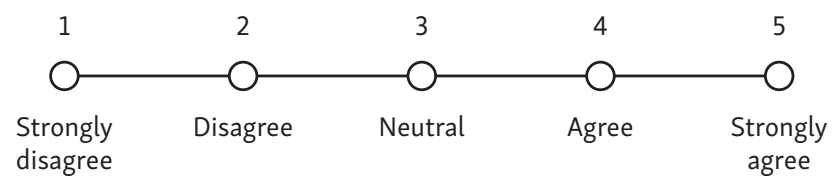

Table 6 - Results of the Pearson correlation coefficient

\begin{tabular}{llll}
\hline & & $\mathrm{X}$ & $\mathrm{Y}$ \\
\hline $\mathrm{X}$ & Pearson Correlation & 1 & $.771^{* *}$ \\
& Sig. (2-tailed) & & .000 \\
$\mathrm{~N}$ & 50 & 50 \\
\hline $\mathrm{Y}$ & Pearson Correlation & $.771^{* *}$ & 1 \\
& Sig. (2-tailed) & .000 & \\
$\mathrm{~N}$ & 50 & 50 \\
\hline
\end{tabular}

\footnotetext{
** Correlation is significant at the 0.01 level (two-tailed)
} 
In Table $6, X$ is the mean score of the participants using the Likert scale and $Y$ is the measurement of overall landmark salience. The results of the Pearson correlation coefficient of landmark salience and the pilot study score means are 0.771 , which can be considered a relatively strong correlation. $\mathrm{P}<0.01$ indicates that the correlation is extremely significant. This experiment verifies that the results of the indoor landmark salience evaluation method proposed above are consistent with the users' spatial cognition.

\section{Experiment 2}

We also wanted to compare the theoretical landmark visual salience values, which were calculated using our proposed method, to the real attraction of these landmarks in a real case scenario. For this purpose, we used the eye-tracking data collected in the second experiment to provide insight on human cognitive processes (Just, Carpenter 1976) and efficiently identify the degree of attention given to specific parts of the surrounding environment.

The second experiment examined the influence on user navigation of different levels of realism in two cartographic visualizations (for more detail see Snopková et al. 2019). Participants were divided into two groups and first had to learn the

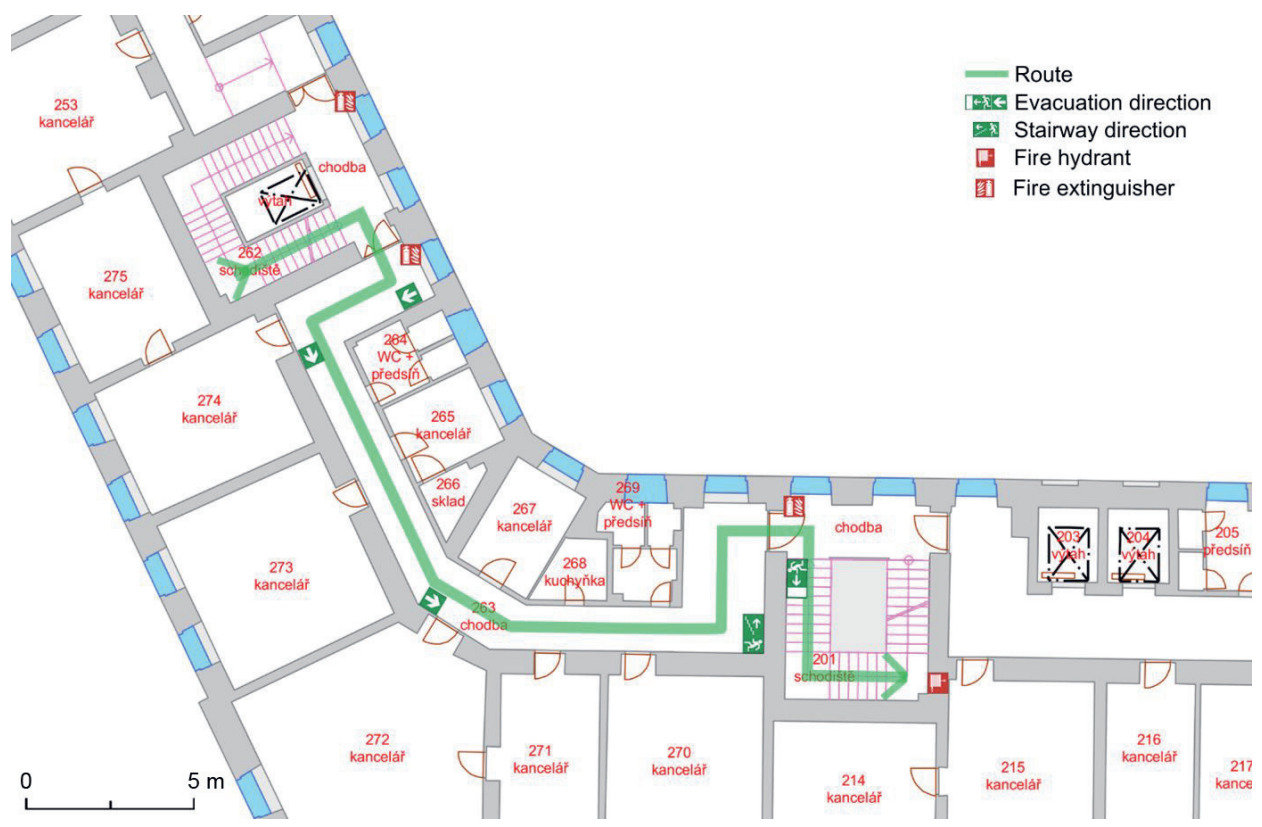

Fig. 6 - Example of schematic floor plan stimuli 
designated evacuation route from either the schematic floor plan (see Fig. 6) or a virtual tour (see chapter 5.2.) before walking along in the real environment (Fig. 6).

Participants were monitored with a mobile eye-tracking device along the route. We were thus able to obtain data about their eye movements, and most importantly, visual fixations on landmarks that they passed along the route. Participants were not familiar with our proposed scoring system for indoor landmark visual salience. The calculated visual salience scores could therefore be objectively compared to the real attention given to landmarks by participants during navigation. Apart from the eye-tracking method, we also asked participants to create their own navigation instructions in order to evaluate the mental spatial representations they developed after learning and walking along the route.

Our method was based on the hypothesis that objects with greater visual salience will have more fixations in the ET data and be mentioned more frequently in the navigation instructions.

\subsection{Participants}

Thirty-six subjects ( 16 females, 20 males) participated in the experiment. More than $80 \%$ of the participants were between 18 and 26 years old. No participants older than 40 years participated in the experiment. Participant were Czechs and Slovaks, mostly university graduates or students and had different work backgrounds.

\subsection{Study area}

The experiment was conducted at the Headquarters of Masaryk University in Brno (Czechia). The 84-metre route led along three floors. The entire route can be found in the virtual tour (used as one of the stimuli in the learning phase) available online at http://ofm.ukb.muni.cz/vt/nav/rektorat/.

\subsection{Methods}

SMI Eye-Tracking glasses $(120 \mathrm{~Hz})$ were used to collect data for the evacuation route. A method commonly used in navigation studies is preparing navigation instructions. After participants learnt the route, they were asked to create navigation instructions. They were asked to imagine that they were instructing a person who had never previously been inside the building. After following the route, 
Table 7 - Results of evaluated overall average landmark salience

\begin{tabular}{llcc}
\hline Salience category & Landmark & Decision point & Overall average salience \\
\hline Most salience & fire extinguisher & 1 & 0.53 \\
& stairs & 4 & 0.53 \\
& green evacuation sign & 4 & 0.53 \\
\hline Medium salience & door & 3 & 0.51 \\
& green evacuation sign & 1 & 0.50 \\
& green evacuation sign & 2 & 0.50 \\
& sign 2nd floor & 3 & 0.50 \\
& sign $2^{\text {nd }}$ floor & 4 & 0.50 \\
& window & 3 & 0.49 \\
& window & 5 & 0.49 \\
& door & 5 & 0.49 \\
\hline \multirow{2}{*}{ Least salience } & stairs & 2 & 0.46 \\
& white door & 2 & 0.44 \\
& radiator & 5 & 0.40 \\
& radiator & 3 & 0.40 \\
& flowers & 5 & 0.36 \\
& flowers & 5 & 0.32 \\
\hline
\end{tabular}
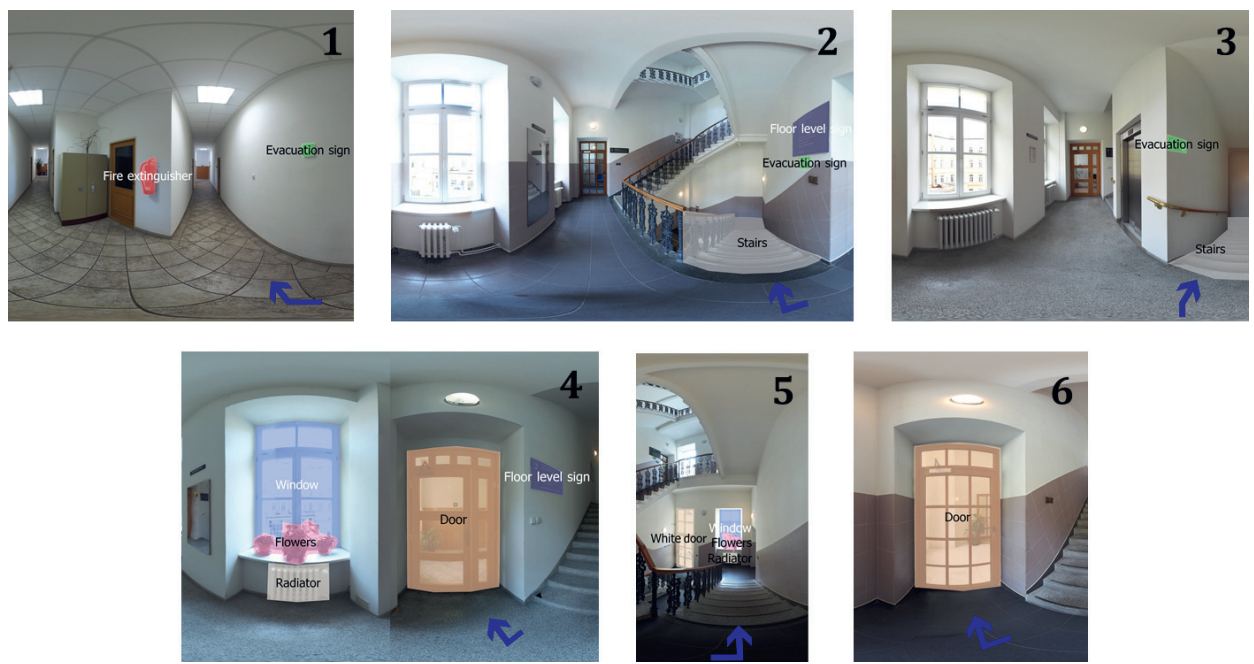

Fig. 7 - Landmarks at decision points in the study area at the Headquarters of Masaryk University (Brno, Czechia)

participants were again asked to prepare instructions so that the effect of actually passing along the route on a participant's created mental spatial representations could be evaluated.

Besides the eye-tracking data and creation of navigation instructions, a set of other methods were used to acquire both quantitative and qualitative data to 
provide better interpretation of the results (Šterrba et al. 2015). Use of these methods influenced the testing procedure and evaluating them was beyond the scope of this paper. We therefore mention them only briefly. An Object-Spatial Imagery and Verbal Questionnaire (Blazhenkova, Kozhevnikov 2008) was used to discover the prevailing cognitive style of participants, as it may have influenced their performance. Participants were asked to sketch the route in a form of a cognitive map (Golledge 1999) while they created navigation instructions. They were also asked to estimate the direction towards the route's end point (before following the route) and the route's start point (after following the route; Okabe, Aoki, Hamamoto 1986). After they followed the route, they were asked some additional questions about how many doors they passed on the way and how many green evacuation signs they noticed. The final task was to determine from the photographs of landmarks whether they had seen the landmark as they followed the route (Münzer et al. 2006).

\subsection{Landmark selection}

Landmarks along the route were selected and their visual salience calculated based on the proposed method. The resulting scores for each landmark are shown in Table 6. In the second experiment, landmarks were mostly represented by the doors, stairs, windows, flowers, fire extinguishers and green evacuations signs and arrows in the university building. (Fig. 7, Table 7)

\subsection{Results}

Recordings of all fixations from the ET data were visualized as an area of interest Sequence Chart, which shows the continuous fixations of each participant on selected area of interests / landmarks. Colour areas show individual fixations and indicate different landmarks. The horizontal axis shows the time each participant spent at decision points. The vertical axis contains all participants. Area of interest sequence charts were created for each decision point. An overview of charts with all decision points is shown in Figure 8.

As mentioned above, detailed area of interest sequence charts of landmarks seen were generated for each decision point. Examples for decision point 3 and 5 are below in Figures 9 and 10 (colour scheme is similar to Figure 8).

Regardless of the two different groups, the eye-tracking data analysis showed the following.

- The greater attention given to functional landmarks similar to the findings of Ohm, Müller, Ludwig (2014) and Viaene et al. (2014) can be confirmed. In this experiment, participants focused especially on doors and stairs. 
- More fixations were counted for objects in the route ahead rather than those behind, as mentioned by Wenczel, Hepperle, Von Stüpnagel (2017).

- Highly focused objects were located at eye level, which may raise their visual salience. It could, however, only mean that these objects were sight anchors, not landmarks (Van Gog et al. 2009). This can happen when participants spend more time fixating on one landmark in the direction of walking. However, objects with lower salience in the direction of walking were not fixated on much or attention was distracted, which supports their given salience.

- Observation cannot confirm whether green arrows or fire extinguishers had higher salience. Based on the data, participants fixated on these objects less than other landmarks. Some reasons may explain this, the first being a significantly smaller visual area, especially for green arrows, than for other landmarks, and second, that eye-trackers are not capable of collecting peripheral vision data (Williams, Davids 1997).

Evaluation of salience categories based on the average number of fixations is most successful in the medium salience category (Fig. 11), which comprises functional landmarks (doors, windows, green arrows and floor signs). Stairs were mainly fixated on in the most salience category, in contrast to green signs and fire extinguishers. In the least salience category, white doors were mostly fixated on, yet flowers and radiators were unattractive to participants, which validates their lower salience. The results for salience categories are shown in Figure 11.

Besides the eye-tracking data, the calculated landmark salience was also compared with the navigation instructions participants devised after passing along the designated route. We hypothesized that the more salience landmarks possessed according to our proposed scoring system, the more participants would mention these landmarks in their navigation instructions.

For more landmarks of the same type (stairs, doors, etc.), it was not possible to distinguish exactly which landmark participants had referred to in their instructions. We therefore compared the cumulative mentions of landmarks in the navigation instructions to their average calculated salience (Fig. 12).

The comparison of landmarks counted in the navigation instructions with their corresponding salience showed some deviations. For example, fire extinguishers were classified as landmarks with the most salience according to our proposed method of landmark salience calculation. However, participants did not mention them very often in their navigation instructions (Fig. 12). This may be because the fire extinguishers were often situated behind doors and participants did not notice them or anticipated that doors were more outstanding and noticeable landmarks, as they also had a functional meaning during navigation. This deviation was confirmed by examining all eye-tracking data recordings and discovering that fire extinguishers attracted a minimum number of fixations. 


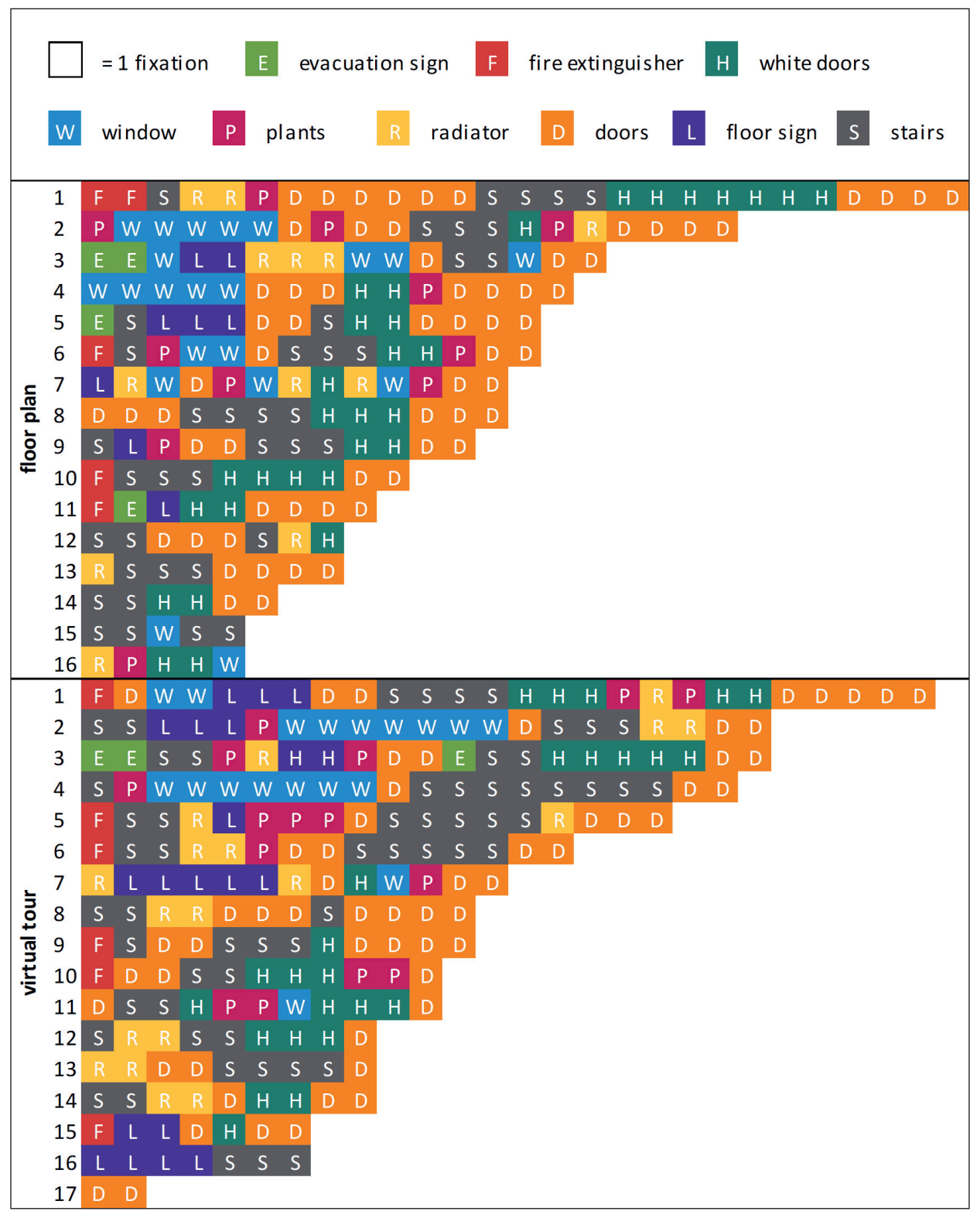

Fig. 8 - Sequence of fixations for each participant (green - green arrow, red - fire extinguisher, grey - stairs, blue - window, pink - flowers, yellow - radiator, orange - doors, dark blue - sign, light brown - white door) 

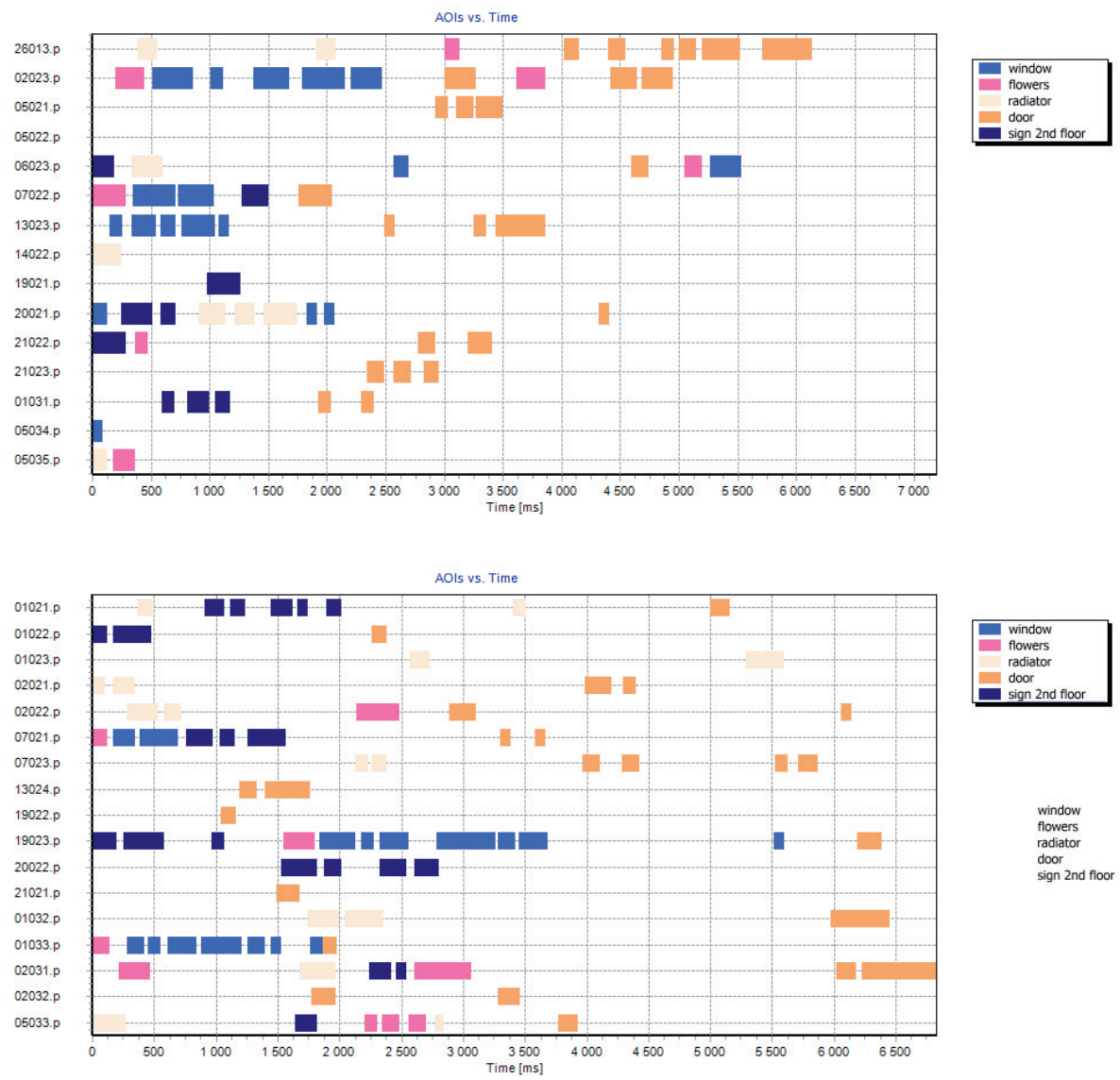

Fig. 9 - Example of detailed area of interest sequence chart for decision point 3 for groups of participants, 2D floor plan (1) and 3D virtual tour (2)

Other deviations concerned white doors, which were classified as landmarks with the least salience, yet participants mentioned them more frequently. These white doors represented alternative escape exits along the designated route, and several participants made a mistake (or almost made a mistake) at this decision point. Here, they had to decide whether to use these white doors to exit the building or proceed through the other door to the right, where the designated route continued. More participants therefore mentioned the white doors in their navigation instructions in order to prevent a visitor from making the (same) navigational mistake.

Participants also mentioned doors and stairs more frequently, as expected based on their visual salience scores. Doors and stairs were repeated landmarks 

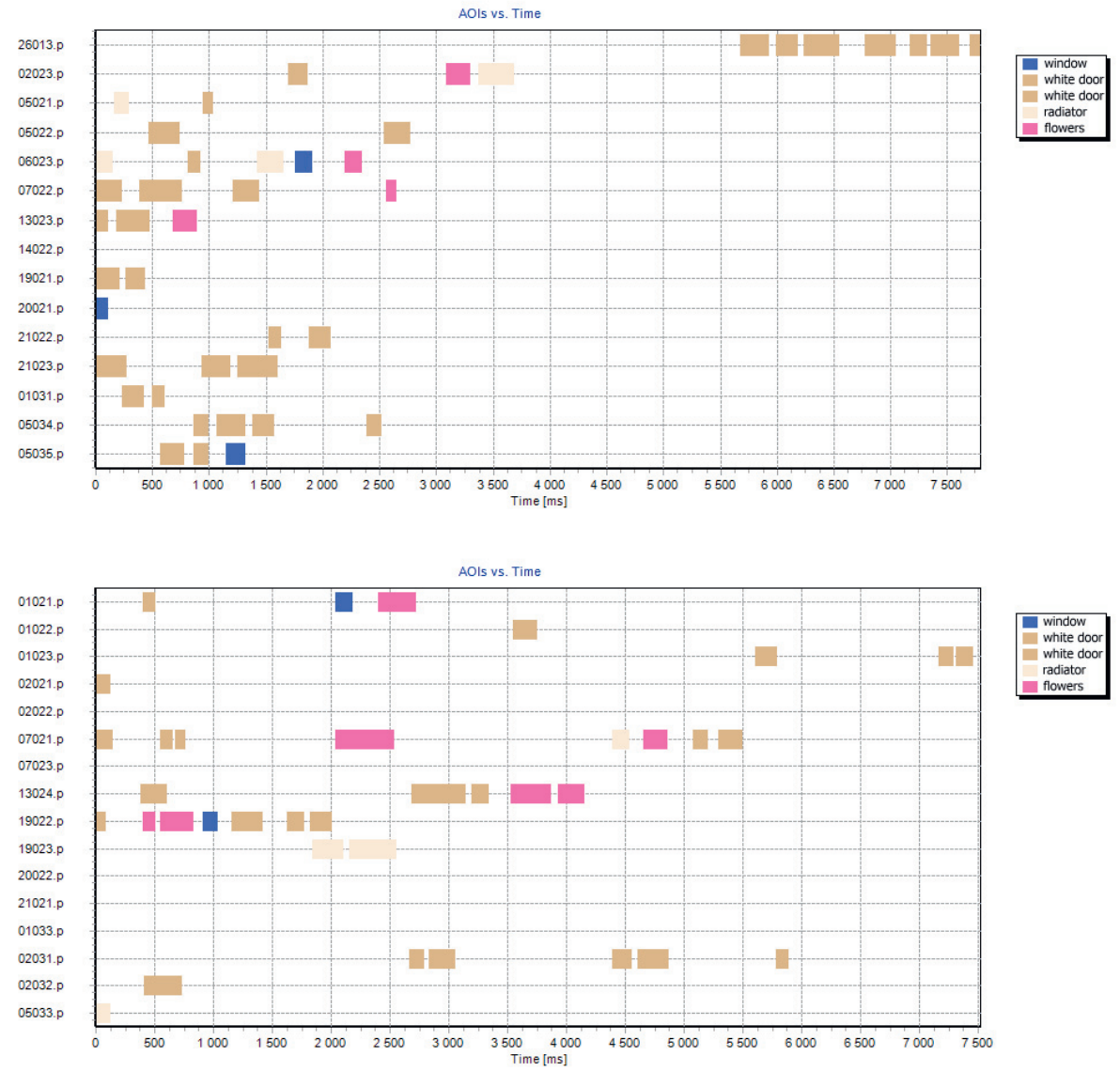

Fig. 10 - Example of detailed area of interest Sequence chart for decision point 5 for groups of participants, 2D floor plan (1) and 3D virtual tour (2)

Fig. 11 - Fixations on landmarks according to salience category

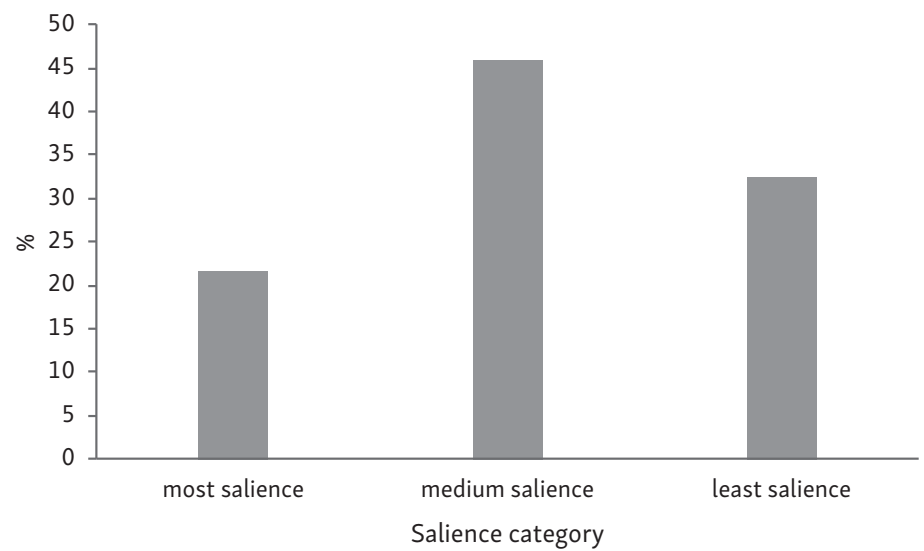




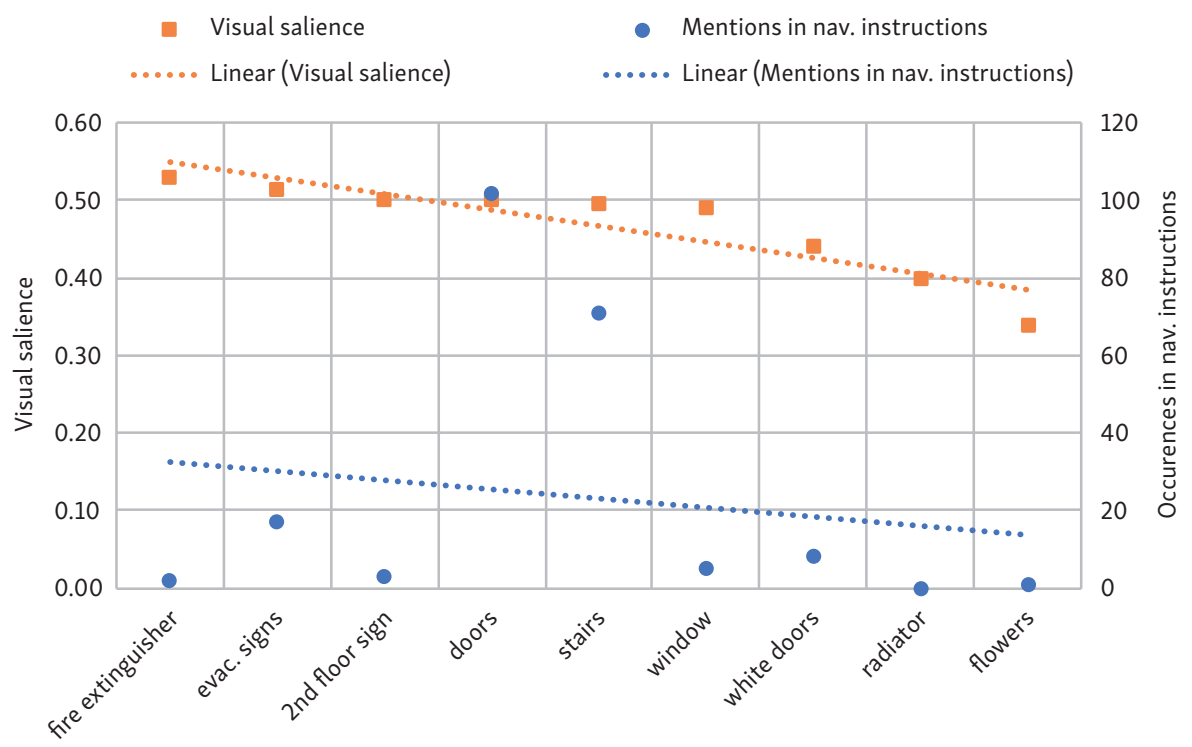

Fig. 12 - Comparison of selected landmark visual salience calculated according to the proposed scoring method and number of mentions of landmarks in navigation instructions created by participants

along the designated route and also had a functional meaning. Therefore, the more numerous mentions of these landmarks in navigation instructions was obvious.

\section{Discussion}

We compared the existing studies in the methods for evaluating and extracting landmark salience, however, these methods were based on computing indicators that were difficult to quantify, and the landmark weighting of each indicator was too arbitrary. We therefore adapted the indicators of indoor landmark salience from Duckham, Winter (2010), Fellner, Huang, Gartner (2017); Lyu, Yu, Meng (2015), who argued that indicators of landmark salience for spatial objects should be evaluated from three aspects: visual, semantic and structural. The aim of this study was to propose a method for an instance-based scoring system in order to compute the indoor landmark salience, and two conduct two field experiments designed to validate the indoor landmark salience evaluation method. For the verification experiments, we applied objective and subjective methods. Experiment 1 used a subjective method consisting of a questionnaire, while Experiment 2 used an objective method consisting of eye-tracking, which showed that this method 
can be used to quantitatively calculate user preferences of indoor landmarks for navigation.

In Experiment 1 the different landmark categories were ranked from high to low: shops, function, information and furniture. The experiment was conducted in a shopping mall; its main landmarks were shops and the functional elements. These two categories played an important role in indoor navigation. The results of this experiment were consistent with the results of Ohm, Müller, Ludwig (2014). However, its main difference was that that shops exceeded function. The functional landmarks were less noticeable because some of the doors were less visible. Finally, thirty subjects were also recruited to participate in the verification experiment by scoring the selected landmarks in the study area using a Likert scale. An analysis of the correlation between the results of the computed overall landmark salience and users' spatial cognition in indoor navigation showed that they were consistent with each other.

Overall, the results obtained from the indoor landmark salience evaluation method proposed in this paper can be considered mostly consistent with the characteristics of indoor landmark types. In Experiment 2, mobile eye-tracking was used in an experiment with a cognitive approach. The recorded data showed that a participant's distribution of attention varied when observing indoor landmarks. The most fixations were recorded on functional elements (doors, windows), which is consistent with the findings of Ohm, Müller, Ludwig (2014) and Viaene et al. (2014), and information (signs indicating floors) landmarks.

\section{Conclusions and future work}

This paper proposed an instance-based scoring system for analysing the indicators (e.g., visual, semantic, structural). An Analytic Hierarchy Process (AHP) was applied to compute the landmark weight in order to evaluate and extract the indoor landmark in navigation. Finally, two experiments were conducted in order to verify the validity of this method.

Experiment 1 was conducted at the Dongchenghui Shopping mall (Nanjing, China). The method used questionnaires concerning the study area to obtain subjective user and expert spatial information about indoor navigation. Verification showed that the results were consistent with the users' spatial cognition. We also selected the Masaryk University Headquarters (Brno, Czechia) as a study area and obtained the objective data of users' visual records from mobile eye-tracking devices to verify the effectiveness of the indoor landmark salience evaluation method. The experiment showed that landmark categories are related to user preferences. Participants paid more attention to functional landmarks (e.g., doors and stairs). In the shopping mall, the participants preferred commercial 
landmarks for wayfinding, while functional landmarks were secondary. However, the University Headquarters is a location without any commercial landmarks and the functional landmarks were more interesting to the participants. The results calculated according to the indoor landmark salience evaluation method proposed in this paper were verified as mostly consistent with the characteristics of indoor landmark categories. The weight of landmark salience using an analytic hierarchy process is more scientific and rational than the traditional method.

The indoor landmark salience evaluation method combined with the selection of user scoring system was proposed in this paper based on the previous research. It made the evaluation process as simple and convenient as possible. However, the standardization and objectivity of this method still requires further research and improvement. Some indicators also relate to the subject of spatial cognitive differences. Future research can explore the method in different indoor environments.

\section{References}

BLAZHENKOVA, O., KOZHEVNIKOV, M. (2008): The New Object-Spatial-Verbal Cognitive Style Model: Theory and Measurement. Applied Cognitive Psychology, 23, 5, 638-663, doi: 10.1002/acp.147.

CADUFF, D., TIMPF, S. (2008): On the assessment of landmark salience for human navigation. Cognitive processing, 9, 4, 249-267.

DUCKHAM, M., WINTER, S., ROBINSON, M. (2010): Including landmarks in routing instructions. Journal of Location Based Services, 4, 1, 28-52.

ELIAS, B. (2003): Extracting landmarks with data mining methods. In International Conference on Spatial Information Theory. Springer, Berlin, Heidelberg, 375-389.

FELLNER, I., HUANG, H., GARTNER, G. (2017): “Turn Left after the WC and Use the Lift to Go to the $2^{\text {nd }}$ Floor" - Generation of Landmark-Based Route Instructions for Indoor Navigation. ISPRS International Journal of Geo-Information, 6, 6, 183.

GOLLEDGE, R.G. (1999): Wayfinding behaviour. Cognitive mapping and other spatial processes. The John Hopkins University Press, Baltimore, MD.

HÖLSCHER, C., BRÖSAMLE, M. (2007): Capturing indoor wayfinding strategies and differences in spatial knowledge with space syntax. In $6^{\text {th }}$ International Space Syntax Symposium, 043.01-043.12.

HUANG, H., GARTNER, G. (2010): A survey of mobile indoor navigation systems. In: Cartography in Central and Eastern Europe. Springer, Berlin, Heidelberg, 305-319.

HUND, A.M. (2016). Visuospatial working memory facilitates indoor wayfinding and direction giving. Journal of environmental psychology, 45, 233-238.

JUST, M.A., CARPENTER, P.A. (1976): Eye Fixations and Cognitive Processes. Cognitive Psychology, 8, 4, 441-480.

KATO, Y., TAKEUCHI, Y. (2003): Individual differences in wayfinding strategies. Journal of Environmental Psychology, 23, 2, 171-188.

KLEPEIS, N.E., NELSON, W.C., OTT, W.R., ROBINSON, J.P., TSANG, A.M., SWITZER, P., ENGELMANN, W.H. (2001): The National Human Activity Pattern Survey (NHAPS): 
a resource for assessing exposure to environmental pollutants. Journal of Exposure Science and Environmental Epidemiology, 11, 3, 231.

KLIPPEL, A., WINTER, S. (2005): Structural salience of landmarks for route directions. In: International Conference on Spatial Information Theory. Springer, Berlin, Heidelberg, 347-362.

LI, L., MAO, K., LI, G., WEN, Y. (2018): A Landmark-based cognition strength grid model for indoor guidance. Survey Review, 50, 361, 336-346.

LI, X., WU, X.Q., YIN, Z.H., SHEN, J. (2017): The influence of spatial familiarity on the landmark salience sensibility in pedestrian navigation environment. International Archives of the Photogrammetry, Remote Sensing \& Spatial Information Sciences, 42, 2, 83-89.

LORENZ, B., OHLBACH, H.J., STOFFEL, E.P. (2006): A hybrid spatial model for representing indoor environments. In International Symposium on Web and Wireless Geographical Information Systems. Springer, Berlin, Heidelberg, 102-112.

LYNCH, K. (1960): The image of the city, 11.

LYU, H., YU, Z., MENG, L. (2015): A Computational Method for Indoor Landmark Extraction Progress in Location-Based Services 2014, Springer, 45-59.

MILLONIG, A., SCHECHTNER, K. (2007): Developing landmark-based pedestrian-navigation systems. IEEE Transactions on intelligent transportation systems, 8, 1, 43-49.

MÜNZER, S., ZIMMER, H., SCHWALM, M., BAUS, J., ASLAN, I. (2006): Computer Assisted Navigation and the Acquisition of Route and Survey Knowledge. Journal of Environmental Psychology, 26, 300-308.

NOTHEGGER, C., WINTER, S., RAUBAL, M. (2004): Selection of salient features for route directions. Spatial cognition and computation, 4, 2, 113-136.

OHM, C., MÜLLER, M., LUDWIG, B. (2015): Displaying landmarks and the user's surroundings in indoor pedestrian navigation systems. Journal of Ambient Intelligence and Smart Environments, 7, 5, 635-657.

OKABE, A., AOKI, K., HAMAMOTO, W. (1986): Distance and Direction Judgment in a LargeScale Natural Environment: Effects of a Slope and Winding Trail. Environment and Behavior, 18, 755-772.

PETERS, D., WU, Y., WINTER, S. (2010): Testing landmark identification theories in virtual environments. In International Conference on Spatial Cognition. Springer, Berlin, Heidelberg, 54-69.

PRESSON, C.C., MONTELLO, D.R. (1988): Points of reference in spatial cognition: Stalking the elusive landmark. British Journal of Developmental Psychology, 6, 4, 378-381.

RADOCZKY, V. (2003): Cartographic support of route descriptions for pedestrian navigation systems. Completion Thesis, Vienna University of Technology.

RAUBAL, M. (2001): Human wayfinding in unfamiliar buildings: a simulation with a cognizing agent. Cognitive Processing, 2, 3, 363-388.

RAUBAL, M., EGENHOFER, M.J. (1998): Comparing the complexity of wayfinding tasks in built environments. Environment and Planning B: Planning and Design, 25, 6, 895-913.

RAUBAL, M., WINTER, S. (2002): Enriching wayfinding instructions with local landmarks. In International conference on geographic information science. Springer, Berlin, Heidelberg, 243-259.

RICHTER, K.F., WINTER, S. (2014): Landmarks: GIScience for Intelligent Services. Springer Publishing Company, Incorporated.

RUSSO, D., ZLATANOVA, S., CLEMENTINI, E. (2014, November): Route directions generation using visible landmarks. In: Proceedings of the sixth ACM SIGSPATIAL international workshop on indoor spatial awareness, ACM, 1-8. 
SAATY, T.L. (1994): How to make a decision: the analytic hierarchy process. Interfaces, 24, 6 , 19-43.

SIEGEL, A.W., WHITE, S.H. (1975): The development of spatial representations of large-scale environments. In Advances in child development and behaviour, 10, 9-55.

SNOPKOVÁ, D., ŠVEDOVÁ, H., KUBÍČEK, P., STACHOŇ, Z. (2019): Navigation in Indoor Environments: Does the Type of Visual Learning Stimulus Matter? ISPRS International Journal of Geo-Information, 8, 6, 1-26, doi: 10.3390/ijgi8060251.

SORROWS, M.E., HIRTLE, S.C. (1999): The nature of landmarks for real and electronic spaces. In International Conference on Spatial Information Theory. Springer, Berlin, Heidelberg, 37-50.

ŠTĚRBA Z., ŠAŠINKA, Č., STACHOŇ, Z., ŠTAMPACH, R., MORONG, K. (2015): Selected Issues of Experimental Testing in Cartography. MU Brno.

VAN GOG, T., KESTER, L., NIVELSTEIN, F., GIESBERS, B., PAAS, F. (2009): Uncovering cognitive processes: Different techniques that can contribute to cognitive load research and instruction. Computers in Human Behavior, 25, 2, 325-331, doi:10.1016/j.chb.2008.12.021.

VIAENE, P., OOMS, K., VASTEENKISTE, P., LENOIR, M., E MAEYER, P. (2014): The use of eye tracking in search of indoor landmarks. Proceedings of the $2^{\text {nd }}$ International Workshop on Eye Tracking for Spatial Research, ET4S 2014, Vienna, 52-56.

WENCZEL, F., HEPPERLE, L., VON STÜPNAGEL, R. (2017): Gaze behavior during incidental and intentional navigation in an outdoor environment, Spatial Cognition \& Computation, 17, 1-2, 121-142, doi: 10.1080/13875868.2016.1226838.

WILLIAMS, A.M., DAVIDS, K. (1997): Assessing cue usage in performance contexts: A comparison between eye-movement and concurrent verbal report methods. Behavior Research Methods, 29, 3, 364-375.

WINTER, S., TOMKO, M., ELIAS, B., SETER, M. (2008): Landmark hierarchies in context. Environment and Planning B: Planning and Design, 35, 3, 381-398.

YANG, L., WORBOYS, M. (2011): A navigation ontology for outdoor-indoor space:(work-inprogress). In Proceedings of the $3^{\text {rd }}$ ACM SIGSPATIAL international workshop on indoor spatial awareness, ACM, 31-34.

ZHANG, X., LI, Q., FANG, Z. (2010): An approach of generating landmark chain for pedestrian navigation applications. Geomatics and Information Science of Wuhan University, 35, 10, 1240-1244.

\section{ACKNOWLEDGMENTS}

The article was funded by the grant of the National Key R\&D Program of China (2016YFE0131600), "Dynamic mapping for risk and crises management in big data era" and NSFC (National Natural Science Foundation of China) project No. 41871371, "Indoor navigation map generalization model and methods considering the constraint of hierarchical landmarks", and the grant of the Ministry of Education, Youth and Sports of the Czech Republic (grant number LTACH-17002) Dynamic mapping methods oriented to risk and disaster management in the era of big data. Dajana Snopková was also supported by a grant of the Masaryk University No. MUNI/A/1576/2018 called "Complex research of the geographical environment of the planet Earth".

The content of this Special Issue of Geografie Journal has been prepared together with the International Cartographic Association, namely with the Commission on Cognitive Issues in 
Geographic Information Visualization, the Commission on Use, User and Usability Issues, and the Commission on Atlases. Selected authors were contacted during the ICA Commissions' joint workshop "Atlases, Cognition, Usability" held in Olomouc (Czechia) at Palacký University in April 2018. The content of the Special Issue reflects the current topics handled by the Commissions and we hope that the special issue increases awareness and knowledge of cognitive and usability issues with geographic information among the broader geographic community. Preliminary selection of manuscripts and pre-submission communication with authors was done by Petr Kubíček (Masaryk University, proposed incoming co-Chair of the Cognitive Issues in Geographic Information Visualization Commission) and Amy Griffin (RMIT University, current co-chair of the Cognitive Issues in Geographic Information Visualization Commission). We thank all of the authors who contributed their research to this special issue. 